\title{
ORTOGRAFÍA ESPAÑOLA: ESTRATIFICACIÓN SOCIAL Y ALTERNATIVAS*
}

\section{ANTECEDEnTES}

Mi interés por el análisis de la ortografía española data de unos pocos años ${ }^{1}$. A partir de lo que la lingüística actual me había ofrecido, partía yo del principio de que el objeto de estudio era fundamentalmente la lengua hablada. Afortunadamente la filología - otra de mis tradiciones - me permitió incluir ese terna en mis preocupaciones. Sin embargo, no traté de salvar los supuestos o imaginarios obstáculos académicos sin motivo: mis inquietudes tenían una razón.

Todo empezó cuando tuve la oportunidad de escribir textos escolares para niños de primaria ${ }^{2}$. Allí me fue necesario reflexionar sobre las normas ortográficas del español - sus razones y sinrazones - para tratar de encontrar un sentido a su enseñanza. Más adelante me fue posible - junto con la ayuda de muchas otras personas - recoger una muestra a nivel nacional de textos con tema libre escritos por niños de tercero a sexto grado de primaria, con edades que van de $\operatorname{los} 9$ a los 13 años. Ese corpus es la base del proyecto "Análisis de textos infantiles", que se de-

* Este trabajo fue leído en el Segundo Taller Internacional sobre el Español en América, La Habana, Cuba, 8 al 12 de mayo de 1989. La versión actual se ha modificado y ampliado.

1 Terminé la primera de estas investigaciones en 1984, cuando presenté en Santo Domingo, República Dominicana, una ponencia sobre la ortografía en tercer grado de primaria (Ávlla 1984a).

${ }^{2}$ Fui editor y coautor de los libros de texto gratuitos para las escuelas primarias de México (Ávila et al. 1980, 1981, 1982). Previamente había colaborado como coautor en los libros de texto de las ediciones de 1972 a 1974 .

${ }^{3}$ La muestra fue diseñada por la Secretaría de Educación Pública de México, y fue recopilada por los maestros y demás autoridades de primaria. Para una versión detallada del proyecto y de la recopilación y la confiabilidad 
sarrolla en el Centro de Estudios Lingüísticos y Literarios de F Colegio de México.

Los resultados que obtuve del análisis del corpus - cuyos dé tos completos para la primaria mexicana presento ahora- $\mathrm{m}$ llevaron a reflexionar sobre la importancia que se le da a la ortc grafía frente a la necesidad de lograr una alfabetización más ef ciente de niños y adultos, por una parte, y la importancia de lc grar una mayor calidad en la redacción por otra. Me referiré todo esto a continuación, así como a la posibilidad de intenté una simplificación de las normas ortográficas actualmente en use

\section{LA ORTOGRAFÍA EN LA ESGUELA PRIMARIA: PROCEDIMIENTO E HIPÓTES}

Para el análisis del corpus mediante el empleo de computadora desarrollamos programas específicos que nos permitieron estı diar los textos infantiles en dos grandes aspectos: el lenguaje y contenido $^{4}$. La investigación que ahora presento se ubica dent del estudio del lenguaje -o, más específicamente, de su tran cripción gráfica. Abarca la totalidad de los 4047 textos de muestra ${ }^{5}$.

de la muestra, cf. Ávila 1988. Véase también Ávila 1984b. En el proyer han participado Elsa Pallavicini y Aralia López, quienes diseñaron las bas del análisis del contenido de los textos. Más adelante colaboró Octavio Rive en el arduo trabajo de la codificación y la revisión de los datos por computad ra. Una descripción pormenorizada de todo esto aparece en VázQuez 198 Allí también se incluye un análisis de la temática de los textos infantiles. U: selección de textos de esos niños - aunque no incluida en la muestra- apar ce en ÁviLA 1982.

${ }^{4}$ El contenido de los textos infantiles se ha analizado en las tesis de $\mathrm{R}$ DRíguez López 1986 y de VázQuez 1989 (véase además n. anterior). Por ot parte, está en elaboración la tesis de Evelyn Arizpe, quien investiga el proce de emisión (por parte de los autores) y de recepción (por parte de los niños) cuentos infantiles y considera, entre otros aspectos, el nivel de comprensi del texto con base en los datos del proyecto. En cuanto al estudio del lengua por ahora se ha investigado el léxico (Ávila 1986a). Por otra parte, se ha c sarrollado - en colaboración con José Antonio Delgado, de El Colegio de $\mathrm{N}$. xico- el programa de cómputo ARCO (Autorregulación por Computador para simular al lector infantil y lograr así una comunicación escrita ir eficiente con los niños. Mediante ese programa actualmente revisamos las c fimiciones de los diccionarios infantiles que estamos redactando y en cuya e boración han participado Sara Giambruno y Evelyn Arizpe, de El Colegio México, y María Ruiz, de la Universidad Pedagógica Nacional.

${ }^{5}$ Previamente presenté los resultados que obtuve para los fonemas / 
Los documentos originales de la muestra, escritos a mano por los niños, fueron retranscritos también manualmente por nosotros para que fueran fácilmente leídos por quienes los procesaron por computadora ${ }^{6}$. Nuestra retranscripción fue textual pero, al mismo tiempo, se añadió una notación mediante la cual más adelante se pudo hacer el recuento de los errores ortográficos - a los que llamo lapsogramas- y los aciertos -u ortogramas. La notación, básicamente, incluye tres tipos de lapsogramas:

$$
\begin{aligned}
& \text { sustitución: } \quad \mathrm{X}=\mathrm{Y}(\mathrm{ej} .: \operatorname{tra} b=\text { vieso, enton } s=c \mathrm{es}) \text {; } \\
& \text { adición: }>\mathrm{X} \text { (ej.: >había, a }>\text { hora); y } \\
& \text { supresión: } \mathrm{X}<\text { (ej.: rr<atón, } h<\text { ojalá). }
\end{aligned}
$$

Las características fonológicas generales del español de México - seseante y yeísta- determinaron en buena medida la selección de los fenómenos que se analizarían. De esta manera, a partir de la versión que llamamos fuente ${ }^{7}$, se recogieron las variantes ortográficas o alogramas correspondientes a la letra $\langle\mathrm{h}\rangle$ y a los fonemas $/ \mathrm{b} /, / \mathrm{j} / /^{8} / \mathrm{y} /{ }^{9} \mathrm{y} / \mathrm{s} /$ (tabla 1 , en el apéndice).

Por otra parte, de acuerdo con las variables más significativas

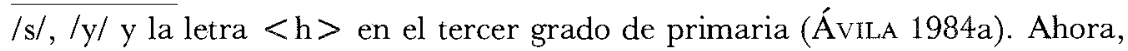
además de extender la investigación hasta el sexto grado, incluyo el fonema /j/. Naturalmente, hay otros casos de lapsogramas, como los que corresponden, por ejemplo, a los fonemas $/ \mathrm{k} /, / \mathrm{rr} / \mathrm{y}$ al uso del acento gráfico o tilde. No los incluí en esta ocasión porque los dos primeros son muy poco frecuentes, y el último tiene una frecuencia muy alta. Esto sesgaba el análisis de mis datos, que se limitan a los lapsogramas de frecuencia media.

${ }^{6}$ Para todo esto contamos con la muy valiosa ayuda del Departamento de Sistemas del Centro de Procesamiento de Datos Arturo Rosenblueth, de la Secretaría de Educación Pública de México. Ellos hicieron también los programas de cómputo que nos permitieron obtener los resultados en los que se basa esta investigación.

${ }^{7}$ Como señalé supra, la versión fuente corresponde a la retranscripción que hicimos nosotros. La notación que utilizamos, además de indicar los lapsogramas, nos permite, mediante programas de cómputo, producir otras dos versiones: la infantil, que genera los textos tal como los escribieron los niños y nos sirve para constatarla con los originales de la muestra; y la normalizada, en la cual se transcriben los textos sin errores y ortográficos y que utilizamos para estudiar el léxico u otros aspectos lingüísticos de los niños.

${ }^{8}$ Transcribo así el fonema $/ \mathrm{x} /$ por razones prácticas de uso y porque, además, así podría escribirse si se hiciera una reforma ortográfica basada en un alfabeto fonológico práctico como la que he propuesto: véanse ÁviLA 1986 b y 1989.

${ }^{9}$ Se incluyó aquí la letra $y$ final de palabra, que fonológicamente corresponde, en la mayoría de los casos, al fonema /i/. 
de la muestra ${ }^{10}$, los alogramas fueron analizados por grado e colar $\left(3^{\circ}, 4^{\circ}, 5^{\circ}\right.$ y $6^{\circ}$ de primaria), sexo, zona (urbana, rural y nivel socioeconómico (alto, medio, bajo). Las hipótesis son li siguientes:

1. Habrá más lapsogramas en tercero que en los demás gri dos de la primaria, y su cantidad descenderá conforma se avanz de uno a otro grado, ya que es de suponerse que a mayor escoli ridad mayor será el aprendizaje de la ortografía.

2. Las niñas tendrán menos lapsogramas que los niños, pc razones culturales: ellas son, en general, menos agresivas y está más orientadas a las demandas escolares ${ }^{11}$. Es probable asimi mo que haya motivos biológicos que expliquen el hecho de qu las representaciones mentales de las niñas parecen sustentar: más en procesos lingüísticos que las de los niños ${ }^{12}$.

3. Los alumnos de escuelas ubicadas en las ciudades tendrá menos lapsogramas que los que viven en el campo. La hipótes se fundamenta en la consideración de que los niños de la ciuda

10 Véanse para esto Ávila 1985 y 1988.

11 De acuerdo con Gardner (1978, p. 212), "Boys tend to be more mu cular and physically active than girls and to perform better than girls at visu and spatial tasks. Girls may be less agressive and more gifted verbally" (véa también n. siguiente). Por otra parte, Terman \& Tyler (1954, pp. 106 1114) en la aplicación del Stanford Achievement Test a millares de niños enco traron que las niñas tuvieron una puntuación significativamente más alta q1 los niños en ortografía y uso del lenguaje. El instrumento empleado (SAT) uno de los más ampliamente utilizados para medir el rendimiento académi de los niños estadounidenses. En mis propias investigaciones advertí asim: mo que las niñas de tercer grado de primaria poseían una mayor cantidad . léxico que los niños (véase ÁviLA 1986a).

12 Las niñas - señalan GARdNER et al. (1975, pp. 13-21)- tienden modificar los estímulos ambientales mediante recursos lingüísticos, lo que refleja en su mayor habilidad para recuperar la información lingüística post riormente (puesto que así la almacenaron). En una investigación posteric GARDNER (1978, p. 212; véase además $n$. anterior) considera que puede $h$ ber alguna determinación biológica que incida en el comportamiento vert de niños y niñas, la que es reforzada por los roles sociales: "Because the trends, however caused, are routinely reinforced by society, each individu receives a gentle push toward the sex role expected by society". Sin embarg este tipo de causas aún están en discusión. Como señala SHapiro (1990, 55), quien ofrece un panorama resumido pero suficientemente extenso y a tualizado de la cuestión, "boys learn to read more slowly than girls, for i stance, and suffer more reading disabilities such as dyslexia", aunque aña más adelante que "No matter where they stand on the question of biolo: versus culture, social scientists agree that the sexes are much more alike th: they are different". 
tienen mayor contacto con la lengua escrita, y se les demanda un mayor uso de ella; los niños rurales, en cambio, tienen una cultura que, por sus características, se apoya más en el uso oral del lenguaje y tienen menos necesidad de emplear el lenguaje escrito.

4. Se encontrarán menos lapsogramas en los textos de los niños de nivel socioeconómico alto que en los de los niveles medio y bajo. En los grupos privilegiados, dado que tienen la posibilidad de hacerlo, se valora más la lengua escrita y, en general, se facilita a los niños la adquisición de conocimientos ${ }^{13}$.

\section{ORTOGRAFÍA EN PRIMARIA: RESULTADOS}

Comentaré a continuación los resultados que obtuvimos en relación con cada una de las categorías de alogramas en el total de textos de la muestra. Más adelante une referiré a los porcentajes de lapsogramas por grado escolar y por las variables sexo, zona y nivel socioeconómico.

Para el total de la muestra -que, como indiqué, abarca 4047 textos- se leyeron por computadora 426700 alogramas, de los cuales el $6.57 \%$ fueron lapsogramas, y ortogramas el complementario $93.43 \%$ (tabla 2 y gráfica 1). En cuanto a las cinco clases o categorías de alogramas analizados, los porcentajes de lapsogramas -en orden descendente de frecuencias - fueron: 1) letra $\langle\mathrm{h}\rangle, 22.38 \%$; 2) fonema $/ \mathrm{b} /, 11.90 \%$; 3) fonema $/ \mathrm{j} /$, $5.87 \%$; 4) fonema $/ y /, 4.72 \%$; y 5 ) fonema $/ \mathrm{s} /, 3.55 \%$.

No es sorprendente que la $\langle\mathrm{h}\rangle$ produzca el mayor número de lapsogramas. Ya lo había advertido previamente, cuando hice el mismo análisis en los textos tercero de primaria ${ }^{14}$. En esa ocasión di varias razones para explicarlo, las cuales se pueden resumir en la inconsistencia de las normas académicas ${ }^{15}$. Motivos

${ }^{13}$ Pueden recordarse, con todas las limitantes que han puesto sus críticos, las conocidas tesis de B. Bernstein $(1971,1974)$. Por otra parte, Brown 1985 -quien también tiene muchas objeciones a los planteamientos de Bernstein- coincide con él y con lo que yo señalo en cuanto a que los estratos altos tienden a facilitar la adquisición de conocimientos a los niños (tomé la referencia de Brown de una versión previa a la publicación de su artículo). En lo que respecta a la redacción infantil, BALDI (1972, p. 377) confirma explícitamente las tesis de Bernstein en niños italianos de 9 a 10 años.

${ }^{14}$ Cf. Ávila 1984a y 1986b, p. 12.

${ }^{15}$ Como señala Alcalá (1983, p. 103), "el hablante, para usar 'correctamente' una letra que no tiene sonido [...] debe atender a nueve apartados o reglas diferentes que a su vez contienen una maraña de usos contradictorios. 
semejantes se pueden aducir para la alta frecuencia de lapsogr mas en la escritura de los fonemas $/ \mathrm{b} / \mathrm{y} / \mathrm{j} /{ }^{16}$.

Por otra parte, los lapsogramas correspondientes a los fon mas $/ y / y / s /$, a pesar de que afectan a los niños mexicanos seantes y yeístas - tuvieron los porcentajes más bajos de todc De estos, el más bajo correspondió al fonema /s/. Las diferenci porcentuales entre los lapsogramas de este fonema y los de /j/ /b/ son claramente significativas. El relativo buen manejo de transcripción del fonema /s/ puede deberse, por una parte, a $\mathrm{q}$ las reglas para el uso de $z$ y $c$ son complementarias y permit oponer esas dos letras a la $s$ y su empleo. También puede sur nerse que se pone especial cuidado en su enseñanza.

En lo que se refiere a los grados de primaria, como se pla teaba en la hipótesis, los porcentajes de lapsogramas desciene conforme los niños avanzan de tercero a sexto. En el tercer gra el porcentaje de lapsogramas es de $8.57 \%$; en el cuarto grac de $7.29 \%$; en el quinto, de $6.80 \%$; y en el sexto de $4.67 \%$ (tab. 3 a 6 y gráfica 2). De la comparación se puede advertir que menor diferencia aparece entre los grados cuarto y quinto, $y$ mayor entre quinto y sexto. En otras palabras, se aprende $n$ ortografía en cuarto grado que en quinto, y más aún en sexto q en el grado anterior. La explicación puede encontrarse, tenta vamente, en el hecho de que en el quinto grado los alumnos tran al último ciclo de la primaria. Esto implica un cambio i portante en los planes de estudio en relación con los del cua grado, que es en parte una continuación y profundización . grado anterior. Frente a esto, el quinto grado incluye mucha formación nueva, cuya asimilación no se consigue plenamente no hasta el último grado de la primaria, en el cual se reiteran nuevos temas con mayor profundidad.

Los porcentajes para las demás variables muestran que las ñas tuvieron $5.66 \%$ de lapsogramas y los niños, $7.79 \%$ (tabl

Y para colmo, en la décima regla [...] la Academia se declara incapaz de denar el caos y lacónicamente termina: «Acerca de otras muchas voces se escriben con $h$, no se pueden dar reglas seguras. Consúltese el Catálogo figura al final de la Gramáticas".

${ }^{16}$ Aparentemente resulta muy alto el porcentaje de lapsogramas de Esta impresión probablemente se apoya en la poca frecuencia general de] nema y de su escritura. Los porcentajes deben evaluarse, para encontre un sentido más claro, dentro del conjunto de sus alogramas que, como ; son poco frecuentes: sus ocurrencias en el total de textos que analizo son : nas 19783 (tabla 2). Los alogramas de /s/, en cambio, llegan a 231718 
y gráfica 3). En cuanto a las zonas, los alumnos de escuelas urbanas alcanzaron un porcentaje de $6.19 \%$ y los de escuelas rurales subieron hasta un $8.08 \%$ (tabla 8 y gráfica 3 ). Por último, los estudiantes de estrato socioeconómico alto produjeron sólo un $2.83 \%$ de lapsogramas, los del estrato medio aumentaron el porcentaje hasta un $6.78 \%$, y los de estrato bajo llegaron al $7.17 \%$ (tabla 9 y gráfica 3 ).

Los resultados confirman las hipótesis. Complementariamente, se puede señalar que las mayores diferencias se presentan entre los alumnos de estrato alto - que tienen menos lapsogramas - frente a los de los estratos medio y bajo. Son también significativos, aunque en menor grado, los bajos porcentajes de lapsogramas que se aprecian en las niñas y en los estudiantes de zonas urbanas en contraste con los de los niños y los de las escuelas rurales, que fueron más altos ${ }^{17}$.

\section{Alternativas}

Los datos que he ofrecido, dada su magnitud numérica, son confiables desde el punto de vista estadístico ${ }^{18}$. Consecuentemente, se puede afirmar que al terminar la escuela primaria los niños mexicanos no logran superar los problemas que presenta la ortografía española. Esto se corrobora con el experimento que hizo Negrete sobre el mismo fenómeno. El investigador - experto en inteligencia artificial - alimentó a una computadora con las nor-

${ }^{17}$ Mis resultados confirman los que obtuve previamente en el análisis del léxico de los alumnos de tercero de primaria (Ávila 1986a). En ese aspecto también las niñas, los alumnos de zonas urbanas y los estudiantes de nivel alto tuvieron más léxico, respectivamente, que los niños, los alumnos de escuelas rurales y los estudiantes de grupos sociales menos favorecidos. En cuanto a la eficiencia en la lectura, APPLEBEE et al. (1988, p. 8) llegan a resultados semejantes en su investigación sobre niños estadounidenses de los grados 3,7 y 11. En los tres grados que analizan, las niñas son mejores lectoras que los niños; y los estudiantes urbanos de estratos altos, mejores que los de estratos bajos. Cabe consignar que entre sus variables incluyen la de origen étnico: en este caso, los blancos son más eficientes que los negros y los hispánicos.

${ }^{18}$ Aunque por esta ocasión no fue posible hacer pruebas específicas de confianza para los análisis de lapsogramas, se puede inferir su validez si se comparan con otras variables lingüísticas de la muestra que sí hemos medido: la muestra y la medida de densidad léxica que aplicamos en el análisis del léxico infantil dio un $\mathrm{p}$ de $5 \%$. 
mas de la ortografía española, y constató que la computadora sć lo pudo resolver "el 26\% de los problemas ortográficos" 19 . Pc eso concluye Negrete que, al terminar la primaria, los alumnc - como no tienen el trasfondo cultural necesario- no logra aprender las reglas ortográficas. Naturalmente, esto no se limit a México. Hay investigaciones de otros países -entre ellos A: gentina, Chile y España- que hacen suponer una situació similar ${ }^{20}$.

Por otra parte, se puede inferir de mis datos sobre las escueli primarias mexicanas que quienes asimilan mejor las normas o tográficas son las niñas de nivel socioeconómico alto que vive en las ciudades, y quienes tienen más problemas son los niños d campo de estrato bajo. La ortografía, en consecuencia, caracte1 za positivamente a unos niños y negativamente a otros. $Y$ es tiene consecuencias sociales y educativas, dada la actitud que tiene en relación con esas normas. O'Dogherty, en una invest gación que hizo con adolescentes que cursaban la secundaria $\mathrm{m}$ xicana - de los grados séptimo a noveno, de 12 a 15 años c edad-, considera que "a mayor índice de reprobación may. será el número de faltas de ortografía"21. Y reitera que los gr pos socialmente menos favorecidos son los que, al escribir, tienc más errores ortográficos. Si no se pierde de vista que quienes ti nen menos lapsogramas son los niños de ambientes sociales pri legiados, se podrá comprender a quiénes favorecen las norm ortográficas y quiénes, en consecuencia, las cultivan y las hace suyas ${ }^{22}$.

Por supuesto, hay alternativas. Cualquiera que se tom pienso, debe tener como punto de partida un cambio de actitı hacia la ortografía. Es necesario situarla en el lugar que le corre ponde y considerar, en lo que respecta a la lengua escrita, q son más importantes sus funciones básicas - leer y escribir

${ }^{19}$ Cf. Negrete 1985 , pp. 68.1. El artículo también se publicó posteriı mente: véase NEGRETE y ARIZA 1987, p. 31.

${ }^{20}$ Cf. Polo 1974, pp. 37 ss.

21 O'Dogherty 1983, pp. 96 ss.

22 Para decirlo con palabras de Miguel de Unamuno, esos grupos soc les - consciente o inconscientemente - tal vez se digan a sí mismos: "Si adoptara una ortografía fonética y sencilla que, aprendida por todos pros y bien, hiciera imposibles las faltas ortográficas, ¿no desaparecería uno de modos de que nos distingamos las personas de buena educación de aquel que no han podido recibirla tan esmerada? Si la instrucción no nos sirve a ricos para que nos diferenciemos de los pobres, ¿para qué nos sirve?', a POLO 1974, p. 336. 
que el manejo adecuado de las normas ortográficas. Un camino a seguir sería enseñar más ortografía; otro, intentar una simplificación de las normas actuales para alfabetizar más eficientemente y promover más la lectura y la redacción.

\section{Más ortografía}

La enseñanza de las reglas académicas podría hacerse de manera más eficiente si se ubican adecuadamente los problemas principales de los alumnos, y los alumnos que tienen los principales problemas. Ciertamente, para esto podría servir mi investigación. Si los profesores saben cuáles son los lapsogramas más frecuentes pueden hacer los ejercicios necesarios para lograr que los alumnos los transformen en ortogramas. Además, pueden apoyarse en las frecuencias de lapsogramas y palabras que los contienen - clasificados por grado y por variable- y enseñarlos a partir de los que más se presentan para eliminar el mayor porcentaje de errores. También pueden hacer concursos regionales y nacionales, transmitirlos por radio y televisión y premiar a los alumnos "memoriosos". Esto no sería novedoso: lo hacen en países de habla inglesa, y hacen bien. Su sistema de escritura - de tipo semiográfico ${ }^{23}$, al igual que el del francés- está muy alejado de la lengua hablada y esto -entre otros problemas- conduce a que hava un mayor número de dislexias ${ }^{24}$ en los niños, y un mayor grado de analfabetismo funcional en los adultos. En cuanto a la escritura de nuestra lengua — de tipo fonográfico; aunque con menor relación entre fonemas y letras que el italiano o el finlandés-, los concursos no tendrían que ser tan frecuentes ni requerirían de tanta difusión.

Otra posibilidad, más cara pero más sensata, es conseguir computadoras y hacer que los chicos escriban sus textos mediante procesadores de palabras. Al final bastará con que pasen un "deletreador" como los que ya hay en el mercado. Así se pueden corregir no sólo los errores ortográficos sino incluso las erratas.

${ }^{23}$ Para una descripción detallada de estos tres tipos de escritura, cf. Alarcos 1968. A grandes rasgos, la escritura semiográfica - como la del inglés y el francés - se sitúa entre la fonográfica - como la del italiano y el finlandés-- y la ideográfica - como la del chino.

${ }^{24}$ Véase Downing y Flores 1975, p. 685. Para un análisis extenso de este problema en Hispanoamérica, véase Bravo 1988, pp. 178 ss. 
Lo que -en todo caso- resultaría más adecuado sería inves tigar el aprendizaje de la ortografía como se investigan las dis lexias o la adquisición de la lengua hablada. De esa maner podrían precisarse sus etapas y los logros de los niños - así com de los adultos analfabetas - conforme a su edad, su sexo y s grupo social. $\mathrm{Y}$ así como no se exige a un niño que maneje toda las reglas fonológicas, sintácticas y semánticas de los adultos tampoco deberían hacérsele exigencias parecidas - que podría resultar absurdas- en lo que respecta a la ortografía.

\section{Más alfabetización}

La lengua - su sintaxis, su fonología, su léxico- la hacemos er tre todos, y la autorregulamos entre todos. La ortografía, e cambio, la hicieron unos pocos y no la han querido modificar pesar de que su simplificación beneficiaría a la mayoría de qui nes escriben en español. En 1960, durante el Tercer Congreso c Academias de la Lengua Española, la Academia Cubana, a tr: vés de Adolfo Tortoló, propuso lo siguiente:

He juzgado que ya sería casi anacrónico, y, desde luego, inoportı no, molestar la ocupada atención de esta docta Asamblea con análisis de argumentos, de uno y otro bando, repetidos hasta la $\mathrm{s}$. ciedad a lo largo de los siglos, y entre los cuales - dicho sea de $\mathrm{p}$ so- en contra de la reforma no he podido hallar jamás ni uno so que merezca ser tomado en consideración.

Explicable es, desde luego, la inclinación a la ortografía etim lógica, en los meritísimos académicos del Diccionario de Autorid des: está de acuerdo con las corrientes ideológicas de su tiempo

Pero más explicable es hoy, y, sobre todo, más justificada, preferencia por la ortografía fonética, que, si en la esfera de 1 ideas responde al anhelo de autenticidad de nuestra época, en práctica sería un aporte de eficacia incalculable a las campañ contra el analfabetismo en que están empeñadas las más de nuє tras naciones, ante la urgencia de que todos los hispanohablant sepan leer y escribir, no solamente para elevar su nivel de vi - que ya sería bastante razón - sino también como medio de pr piciar aún más el acercamiento y la cohesión social entre tod nuestros pueblos.

¿Argumentos?

Uno solo, y muy breve: ir sin más demora a la reforma de la ort grafía es el servicio más eminente que la Academia puede prest 
hoy a la alfabetización, al estudio de la lengua, y, en última instancia, a la integración del mundo hispánico ${ }^{25}$.

Las razones de la Academia Cubana no carecen de fundamento. En lo que toca a la ortografía etimológica, se pueden encontrar argumentos en contra desde el siglo XVII. Cuando en 1630 Gonzalo Correas escribe su ortografía, en la dedicatoria al rey - tras abogar por el principio básico de la escritura alfabética: un sonido para cada letra-, le solicita liberar a la escritura del español de "la esclavitud en que la tienen los que estudian latín" 26. Dos siglos más tarde, en el xIx, Andrés Bello considera que "la etimología es la gran fuente de confusión de los alfabetos de Europa" ${ }^{27}$, y demuestra la inconsistencia del criterio etimológico de la Academia Española.

La necesidad de simplificar la ortografía española para lograr una alfabetización más eficiente parece advertirse por lo menos desde 1609. En ese año Mateo Alemán propugnaba por una reforma ortográfica basada en la relación biunívoca de fonemas y letras entre otros motivos porque así se podría "ayudar a los pobreticos niños" y "ahorrar el dinero que gastan inútilmente sus padres"'28. Argumentos semejantes a los de la Academia Cubana utilizan en 1844 Andrés Bello, la Facultad de Humanidades de la Universidad de Chile y Domingo Faustino Sarmiento. El primero además añade explicaciones para rebatir los supuestos problemas causados por las homografías ${ }^{29}$. Y ahora -en la época de la lingüística, la neurolingüística y la psicolingüística- sigue habiendo razones en el mismo sentido. Alarcos interpreta las faltas de ortografía como el deseo inconsciente del que escribe por mantenerse fiel al principio fonográfico: "donde no hay diferencias fónicas no hacen falta diferencias gráficas"'30. Downing y Flores advierten que en las lenguas en las cuales hay una alta correlación entre fonemas y letras casi no hay problemas en el aprendizaje de la lectura y la escritura. Por eso recomiendan - para reducir el problema de las dislexias- que se use un sola transcripción para cada fonema ${ }^{31}$. Y Ferreiro llega prácticamen-

${ }^{25}$ Academia Cubana 1960, pp. 265-266.

26 Apud Rosenblat 1951, p. xlvii.

27 Bello 1823, p. 79. Véase también Bello 1844, pp. 106-108.

28 Apud Alcalí 1983, p. 89. Véase asimismo Rosenblat 1951, pp. xliv ss.

29 Cf. Bello 1844, pp. 106 ss.

${ }^{30}$ Cf. Alarcos 1968, p. 565.

31 Cf. Downing y Flores 1975, p. 685. 
te a las mismas conclusiones a partir de sus investigaciones sobr el aprendizaje de la lengua escrita. Los niños buscan la lógica d la ortografía - y escriben suponiendo que la tiene. Por eso en s escritura "hay diferencias de letras cuando hay diferencias sonc ras y hay igualdad de letras cuando hay similitudes sonoras". D allí que Ferreiro considere que "la eliminación de las irregulari dades de un sistema es algo de la mayor importancia en la adqui sición del lenguaje (oral y escrito)"'32.

\section{Más Redacción}

Una de las conclusiones que arroja el experimento de Negrete Ariza es que Juanito - el nombre que le pusieron al program de cómputo - no aprende ortografía porque para hacerlo neces ta usar las reglas, y no las usa "porque no redacta"'33. Tal pare ce que Juanito ha caído en un círculo vicioso: no sabe ortografí porque no redacta... Y no redacta porque no sabe ortogrc fía, porque teme usar las palabras de cuya transcripción gráfic no está seguro.

Redactar no equivale a transcribir en el papel lo que uno $d$ ce. No es verdad - como pensaba Juan de Valdés- que uno e: cribe como habla. Frente a la lengua hablada, la lengua escrit es permanente y no volátil, e implica una mayor economía y ez plicitud. Es un ejercicio diferente que obliga a organizar el per samiento y a imaginar un lector en abstracto. Estas característ cas ayudan al desarrollo intelectual de los niños, y lo conduce a superar el lenguaje egocéntrico ${ }^{34}$. Por eso es necesario insist en lo básico y no en lo accesorio, para que no se diga - como $\mathrm{r}$ cuerda Julio Casares- que tal parece que incluso en España a

${ }^{32}$ Ferreiro 1986, pp. 71-74. El niño - continúa Ferreiro- "testimon una fuerte exigencia de coherencia lógica, la misma que [...] emplea pa apropiarse de otros objetos del mundo y que los trabajos de Piaget nos h: permitido hacer observables" (p. 76). No obstante esto y lo que yo he arg mentado supra, Lara da otro tipo de razones basadas en el "deber ser" pa apoyar la ortografía actual. Véase en ese sentido mi extensa réplica a las ide de Lara - y a algunas consideraciones de Ferreiro- en Ávila 1986b.

${ }^{33}$ Negrete y Ariza 1987, p. 31.

${ }^{34}$ Véase Schmid, 1985: El lenguaje escrito, para Piaget y Vigotsky, " la forma más elaborada del lenguaje" y, al mismo tiempo, la "más aleja del lenguaje interno-egocéntrico" (pp. 131-132). De acuerdo con Porcher, escritura, además mantiene una relación intrínseca con el poder: "es [...] lenguaje del poder por excelencia" (apud ScHMId 1985, p. 129). 
gunos desean salvar la ortografía "aunque perezca la gramática", -o su equivalente, la redacción ${ }^{35}$.

El círculo vicioso antes comentado en el cual se encierran quienes tienen errores de ortografía y por eso no redactan se puede romper mediante el principio varias veces mencionado de la escritura alfabética: un sonido para cada letra. Este principio es de honda raigambre hispánica: ya lo sostenían Alfonso el Sabio ${ }^{36}$ en el siglo XIII y Antonio de Nebrija ${ }^{37}$ en el xv; y se mantiene en los siglos posteriores con los filólogos que he citado anteriormente ${ }^{38}$.

\section{SONIDOS Y LETRAS}

Una simplificación de la ortografía española no va contra la tradición, sino al contrario: se apoya en ella. Y no supone romper con la unidad ortográfica del español. Como he escrito en otra parte $^{39}$, la idea es hacer más sencillas las reglas y darles la lógica

${ }^{35}$ Para obtener empleo en España - dice Casares-, en algunas ocasiones los solicitantes deben hacer oposiciones en las que se exige la ortografía. En cambio, se descuida la redacción, "como si fuera indiferente al Estado la capacidad de sus futuros servidores para escribir con claridad y soltura", apud Polo 1974, pp. 521-522.

${ }^{36}$ Léanse para esto las palabras de Ángel Rosenblat (1951): cuando la escritura pasa a ser usada por los juglares, "que transformarán al castellano en lengua literaria, se alejará aún más de los orígenes latinos, se ajustará más a la pronunciación: la escritura juglaresca será escritura para el oído" (p. xiv). Y continúa: "Alfonso el Sabio, que recoge y consagra la poesía de los juglares, va a mantener y fijar la ortografía castellana sobre las mismas bases que habían servido a los juglares: la pronunciación popular' (p. xvi).

${ }^{37}$ En el capítulo V de su Gramática, donde trata de las letras, escribe: "assí tenemos de escrivir como pronunciamos, y pronunciar como escrivimos; por que en otra manera en vano fueron halladas las letras"' (NebriJA 1492, p. 116). Y más adelante anota: "podemos inferir la primera regla de la orthografía castellana: que assí tenemos de escrivir como pronunciamos, y pronunciar como escrivimos"' (p. 131).

${ }^{38}$ Como advierte Quilis (NEBRIJA 1492, p. 43), ese principio "ya lo había defendido en Roma el español Quintiliano [...] y sigue vigente [...], a partir de Nebrija, en toda nuestra tradición gramatical'. Recientemente JEsús Mosterín (1981) publicó un interesante y extenso estudio sobre la ortografía fonémica del español. Coincido en general con sus planteamientos, aunque difiero en algunos detalles. Véanse mis comentarios al respecto en Ávila 1986b, pp. 30 ss. y n. 37.

39 Véanse Avila 1986b y 1989. La nueva ortografía se basaría en los fonemas del diasistema o diafonemas, que he descrito en Ávila 1974. 
que suponen en esas normas a los niños y adultos que empieza a escribir. Sin embargo, para mantener la unidad de la escritur de la lengua habría que mantener las transcripciones de los fon mas /z/ y /ll/, aunque no sean de uso general en los países hisp: nicos. Por lo mismo, sería necesario incluir una grafía para fonema /sh/ que aparece en muchas palabras de origen indoam ricano así como en préstamos de otras lenguas europeas, aunqu tampoco se pronuncie en todas las regiones hispanohablantes.

La proposición supone, además, mantener el alfabeto actu sin suprimir una sola letra, ya que es de uso internacional y su en pleo es necesario para diferentes tipos de ordenaciones, como li que se emplean en bibliotecas y archivos. Sin embargo, a part del alfabeto actual, se requiere delimitar el empleo de las letr: para el español, tal como explico a continuación ${ }^{40}$.

\section{A B $C$ D E F G $H$ I J K L M N O P $Q R$ R T U V.W.X Y (Z)}

a) El alfabeto internacional se utilizará completo para las $\mathrm{c}$ denaciones de archivos, bibliotecas y otros.

b) Las reglas de acentuación se mantendrán como hasta ahor

c) La $G$ siempre se pronunciará con sonido velar sonoro ( $g a_{\text {i }}$ gerra).

d) Como consecuencia, la $U$ siempre tendrá sonido, y se s primirá la diéresis (linguista, deguello).

e) La $\tilde{N}$, aunque no se incluye en el alfabeto internacion: debe seguirse empleando, pero considerada como una $N$ con tile por lo que se alfabetizará al final de la $\mathrm{N}$.

$f$ ) Las letras que aparecen en cursivas $(C, H, Q, V, W, X)$ utilizarán sólo para la escritura de palabras de origen no hispáı co, así como para topónimos y antropónimos.

g) La $H$ se empleará para resolver los pocos casos de homol mias que así lo requieran.

h) La $Z$ se mantiene como única transcripción del fonema i terdental sordo, ya que existe en algunas regiones de Espar̃ consecuentemente no se empleará la letra $c$ antes de $e, i$, cor ahora.

i) La $L L$ (alfabetizada en la $L$ ) se mantiene, ya que aún

${ }^{40}$ El planteamiento que expongo se inspira en la manera en que los 1 landeses tratan el alfabeto latino en relación con las palabras finlandesas y de otro origen. Cf. Aaltio 1963, t. 1, p. 16. 
pronuncia el fonema lateral palatal en zonas reducidas de España y América.

j) Se utilizará además la letra $S H$ para transcribir el sonido palatal sordo continuo que aparece en palabras de lenguas amerindias (mishiote, ishtle) y de otras lenguas europeas (sherif, sham$p u ́$ ), pero se alfabetizará dentro de la $S$ (entre sexo... sí, tal como aparece en algunas enciclopedias del idioma español).

\section{PARA Terminar,}

quiero añadir que el problema de la ortografía preocupa también a los hablantes de otras lenguas. En francés se ha intentado una simplificación, lamentablemente limitada, que difícilmente resolverá los problemas básicos de la transcripción escrita de esa lengua, y cuya discusión ha llegado incluso a los más altos niveles políticos, académicos y educativos ${ }^{41}$. En portugués también se producen documentos oficiales en ese sentido ${ }^{42}$. En cuanto al inglés, la situación es semejante, ya que la ortografía - como la del francés- no es biunívoca con respecto al sistema fonológico (véase $\mathrm{n} .23$ y texto supra). Aunque no se habla de posibles reformas, sí se plantean los problemas del spelling y la improbable relación del buen empleo de las normas ortográficas con la inteligencia, la cantidad de lecturas e incluso con el número de textos escritos. De acuerdo con el subtítulo de un artículo, "Los cientí-

${ }^{41}$ Cf. Conseil Supérieur de la Langue Française 1990. Véase además OLIVIERI 1990, quien señala entre los que se oponen a la reforma de la ortografía francesa a los puristas y académicos, y entre quienes la apoyan a los profesores, sobre todo de los primeros grados escolares. El asunto se ha vuelto de interés internacional: cf. el artículo de UNGEHEUER 1991, en la revista Time. El autor, señala de nuevo, que la mayor parte de académicos y los escritores conservadores se oponen y que, en cambio, favorece las reformas la Asociación de Profesores de Francés. Véase además Greenhouse 1991, quien escribe lo siguiente en The New York Times (pp. 1-2): "The spelling report comes after years of angry debate between purists who say their beautiful language should not be tinkered with and dissenters who complain that French schoolchildren -as well as those who learn French in Belgium, Quebec and Africa- are waisting thousands of hours memorizing illogical spellings when that time could be better spent studying computers and quarks'. Más adelante, el autor recoge al siguiente cita: "The purists are going to scream, but children and foreigners will be happy"; y añade que "The Nationai Association of School Teachers backed the recommendations".

42 Véase el Acordo ortográfico da língua portuguesa 1990. 
fieos, los editores y los profesores de inglés todavía no saben po: qué algunas personas inteligentes fallan en ortografía" 43 .

En Kuanto al español, si se aze una simplifikazión de la orto grafía (i se eskribe komo aora muestro) únikamente lo notaríar los ke ya saben leer i eskribir - pero no los niños ni los adulto: analfabetas. Además, es mui probable ke la mayoría de los alfa betizados esté de akuerdo kon los kambios, pues así el aprendiza je de la lektura y la eskritura sería relativamente senzillo ${ }^{44}$, i po dríamos todos dedikarnos a mejorar la kalidad de la redakzión Admito, sin embargo, ke a muchos de mis lektores -aunke nc tengan problemas para leer esto- no les gustará ber estas línea kon la nueba ortografía. Por otra parte, mis lektores tendrán kı azeptar ke su opinión se basa en la tradizión i en la kostumbre i ke el problema prinzipal para lograr el kambio está en el inkons ziente.

En las pájinas anteriores e tratado de ofrezer argumentos lin guístikos, pedagójikos, soziales, neurolójikos i psikolójikos pari apoyar la nezesidad de modifikar las normas ortográfikas Además, e intentado destakar ke el prinzipio de un sonido par: kada letra, en el kual sustento mi proposizión, tiene una larg: tradizión ispánika ${ }^{45}$. Keda para más adelante la diskusión deta liada de los kambios. Kisiera ke aora, por lo menos, se produje ran algunas dudas razonables: kizás sea kombeniente asumi

43 BECK 1988, p. 48: "Whatever the underlying factors, there is a grow ing awareness - in research, if not in corporate hiring circles- that the abil ity to spell has little to do with basic intelligence. Nor does it correlate wit writing ability". Un gran escritor de lengua inglesa, como F. Scott Fitzgeralc era "notoriously bad speller", y lo mismo le sucedía a Ernest Hemingway.

${ }^{44}$ I kienes no kisieran aprender la nuebas normas podrían rekurrir, d nuebo, a un programa de kómputo: en este kaso podríamos ofrezerles el prc grama ORTOFON (Ortografía fonolójika, en bersiones ispánika i mexike na), ke desarrollamos José Antonio Delgado i yo en El Kolejio de México. (D paso: los antropónimos i topónimos no se modifikan). El programa - a dift renzia de los "deletreadores" o spellers ke se benden en el merkado- en be de korrejir los errores retranskribe de la ortografía aktual a la nueba. Aplik ese programa a esta parte final de mi artíkulo.

45 Tradizión ke, por zierto, permite a Julio Cortázar eskribir en Rayue el relato "Otro suisida", kon una ortografia sorprendentemente parezida a 1 ke yo propongo. La diferenzia es ke en su narrazión emplea lo ke yo llamo । alfabeto ispanoamerikano, en el kual se omiten la $z$ i la $l l$. Por otra parte también suprime la $h$ i la utiliza kongruentemente en lugar de la $c h$. (Reiter ke los nombres, apellidos i topónimos pueden segirse eskribiendo al gust del bautizado, komo menzioné en la n. anterior. Por eso transkribo así "Co: tázar".) 
otra aktitud i balorar más la redakzión ke la ortografía, i kizás sea realmente nezesario konsiderar la posibilidad de simplifikar las normas ortográfikas de la lengua española.

Raúl Ávila

El Colegio de México

\section{APÉNDICE \\ TABLAS Y GRÁFICAS}

TABLA 1

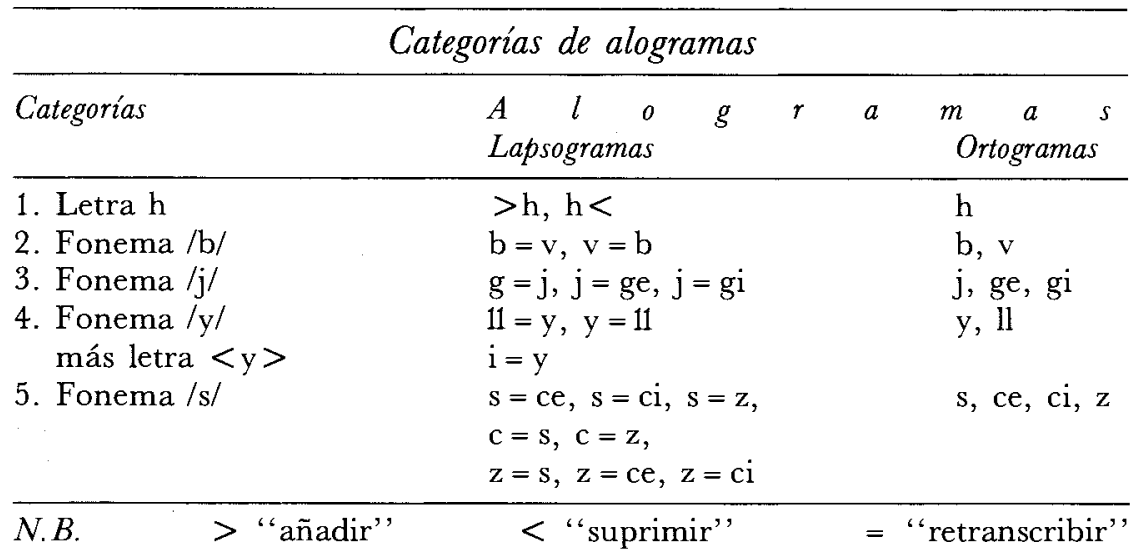

TABLA 2

Alogramas: lapsogramas y ortogramas

Primaria: grados tercero a sexto

\begin{tabular}{lccrcrr}
\hline & \multicolumn{2}{c}{ lapsogramas } & \multicolumn{2}{c}{ ortogramas } & \multicolumn{2}{c}{ alogramas } \\
& frec & \multicolumn{1}{c}{ porcn } & \multicolumn{1}{c}{ frec } & porcn & \multicolumn{1}{c}{ frec } & porcn \\
\hline letra $\mathrm{h}$ & 6817 & 22.38 & 23649 & 77.62 & 30466 & 100.00 \\
fonema $/ \mathrm{b} /$ & 8279 & 11.90 & 61278 & 88.10 & 69557 & 100.00 \\
fonema $/ \mathrm{j} /$ & 1162 & 5.87 & 18621 & 94.13 & 19783 & 100.00 \\
fonema $/ \mathrm{y} /$ & 3552 & 4.72 & 71624 & 95.28 & 75176 & 100.00 \\
fonema /s/ & 8219 & 3.55 & 223499 & 96.45 & 231718 & 100.00 \\
\hline totales & 28029 & 6.57 & 398671 & 93.43 & 426700 & 100.00 \\
\hline
\end{tabular}

núm. de textos 4047 
TABLA 3

Alogramas: lapsogramas y ortogramas

Tercer grado de primaria

\begin{tabular}{lrrrrrr}
\hline & \multicolumn{2}{c}{ lapsogramas } & \multicolumn{2}{c}{ ortogramas } & \multicolumn{2}{c}{ alogramas } \\
& frec & \multicolumn{1}{c}{ porcn } & \multicolumn{1}{c}{ frec } & porcn & \multicolumn{1}{c}{ frec } & porc \\
\hline letra $\mathrm{h}$ & 1387 & 25.02 & 4156 & 74.98 & 5543 & 100.1 \\
fonema $/ \mathrm{b} /$ & 2039 & 15.93 & 10763 & 84.07 & 12802 & 100.1 \\
fonema $/ \mathrm{j} /$ & 320 & 8.06 & 3648 & 91.94 & 3968 & 100.1 \\
fonema $/ \mathrm{y} /$ & 1087 & 7.17 & 14081 & 92.83 & 15168 & 100.1 \\
fonema $/ \mathrm{s} /$ & 1778 & 4.49 & 37838 & 95.51 & 39616 & 100.1 \\
\hline totales & 6611 & 8.57 & 70486 & 91.43 & 77097 & 100.1 \\
\hline
\end{tabular}

núm. de textos 1027

TABLA 4

Alogramas: lapsogramas y ortogramas

Cuarto grado de primaria

\begin{tabular}{lrrrrrr}
\hline & \multicolumn{2}{c}{ lapsogramas } & \multicolumn{2}{c}{ ortogramas } & \multicolumn{2}{c}{ alogramas } \\
& frec & porcn & \multicolumn{1}{c}{ frec } & porcn & \multicolumn{1}{c}{ frec } & porc \\
\hline letra $\mathrm{h}$ & 1719 & 24.48 & 5303 & 75.52 & 7022 & $100 .$. \\
fonema $/ \mathrm{b} /$ & 2098 & 13.31 & 13670 & 86.69 & 15768 & 100. \\
fonema $/ \mathrm{j} /$ & 363 & 7.87 & 4249 & 92.13 & 4612 & 100. \\
fonema $/ \mathrm{y} /$ & 853 & 4.79 & 16926 & 95.21 & 17778 & 100. \\
fonema $/ \mathrm{s} /$ & 1943 & 3.85 & 48544 & 96.15 & 50487 & 100. \\
\hline totales & 6976 & 7.29 & 88692 & 92.71 & 95667 & 100. \\
\hline núm. de textos & 962 & & & & &
\end{tabular}

TABLA 5

Alogramas: lapsogramas y ortogramas

Quinto grado de primaria

\begin{tabular}{lrrrrrr}
\hline & \multicolumn{2}{c}{ lapsogramas } & \multicolumn{2}{c}{ ortogramas } & \multicolumn{2}{c}{ alogramas } \\
& frec & porcn & \multicolumn{1}{c}{ frec } & porcn & frec & porc \\
\hline letra $\mathrm{h}$ & 2096 & 24.78 & 6363 & 75.22 & 8459 & 100. \\
fonema $/ \mathrm{b} /$ & 2393 & 12.25 & 17136 & 87.75 & 19529 & 100. \\
fonema $/ \mathrm{j} /$ & 295 & 5.38 & 5185 & 94.62 & 5480 & 100. \\
fonema $/ \mathrm{y} /$ & 991 & 4.78 & 19758 & 95.22 & 20749 & 100. \\
fonema /s/ & 2501 & 3.70 & 65073 & 93.30 & 67574 & 100. \\
\hline totales & 8276 & 6.80 & 113515 & 93.20 & 121791 & 100. \\
\hline
\end{tabular}

núm. de textos 1024 
TABLA 6

Alogramas: lapsogramas y ortogramas

Sexto grado de primaria

\begin{tabular}{lrrrrrr}
\hline & \multicolumn{2}{c}{ lapsogramas } & \multicolumn{2}{c}{ ortogramas } & \multicolumn{2}{c}{ alogramas } \\
& frec & porcn & \multicolumn{1}{c}{ frec } & porcn & \multicolumn{1}{c}{ frec } & porcn \\
\hline letra $\mathbf{h}$ & 1615 & 17.10 & 7827 & 82.90 & 9442 & 100.00 \\
fonema $/ \mathrm{b} /$ & 1749 & 8.15 & 19709 & 91.85 & 21458 & 100.00 \\
fonema $/ \mathrm{j} /$ & 184 & 3.22 & 5539 & 96.78 & 5723 & 100.00 \\
fonema $/ \mathrm{y} /$ & 622 & 2.90 & 20859 & 97.10 & 21481 & 100.00 \\
fonema $/ \mathrm{s} /$ & 1997 & 2.70 & 72044 & 97.30 & 74041 & 100.00 \\
\hline totales & 6167 & 4.67 & 125978 & 95.33 & 132145 & 100.00 \\
\hline
\end{tabular}

núm. de textos 1034

TABLA 7

\begin{tabular}{|c|c|c|c|c|c|c|c|}
\hline \multicolumn{8}{|c|}{$\begin{array}{l}\text { Alogramas: lapsogramas y ortogramas } \\
\text { Sexos femenino y masculino }\end{array}$} \\
\hline & & \multicolumn{2}{|c|}{ lapsogramas } & \multicolumn{2}{|c|}{ ortogramas } & \multicolumn{2}{|c|}{ alogramas } \\
\hline & & frec & porc & frec & porcn & frec & porcn \\
\hline \multirow[t]{2}{*}{ letra $h$} & feme & 3529 & 20.06 & 14060 & 79.94 & 17589 & $\overline{100.00}$ \\
\hline & mase & 3100 & 25.63 & 8995 & 74.37 & 12095 & 100.00 \\
\hline \multirow[t]{2}{*}{ fon $/ b /$} & feme & 3962 & 10.13 & 35157 & 89.87 & 39119 & 100.00 \\
\hline & mase & 4075 & 14.21 & 24600 & 85.79 & 28675 & 100.00 \\
\hline \multirow[t]{2}{*}{ fon $/ \mathrm{j} /$} & feme & 584 & 5.22 & 10614 & 94.78 & 11198 & 100.00 \\
\hline & mase & 551 & 6.81 & 7540 & 93.19 & 8091 & 100.00 \\
\hline \multirow[t]{2}{*}{ fon $/ y /$} & feme & 1627 & 3.80 & 41165 & 96.20 & 42792 & 100.00 \\
\hline & mase & 1756 & 5.75 & 28788 & 94.25 & 30544 & 100.00 \\
\hline \multirow[t]{2}{*}{ fon $/ \mathrm{s} /$} & feme & 4094 & 3.08 & 128882 & 96.92 & 132976 & 100.00 \\
\hline & mase & 3875 & 4.21 & 88179 & 95.79 & 92054 & 100.00 \\
\hline \multirow[t]{2}{*}{ totales } & feme & 13796 & 5.66 & 229878 & 94.34 & 243674 & 100.00 \\
\hline & mase & 13357 & 7.79 & 158102 & 92.21 & 171459 & 100.00 \\
\hline \multirow[t]{3}{*}{$\overline{\text { núm. txt }}$} & feme & 2283 & & & & & \\
\hline & masc & 1669 & & & & & \\
\hline & total & 3951 & & & & & \\
\hline
\end{tabular}


TABLA 8

Alogramas: lapsogramas $y$ ortogramas Zonas urbana y rural

\begin{tabular}{llrrrrrr}
\hline & & \multicolumn{2}{c}{ lapsogramas } & \multicolumn{2}{c}{ ortogramas } & \multicolumn{2}{c}{ alogramas } \\
& & frec & \multicolumn{1}{c}{ porc } & \multicolumn{1}{c}{ frec } & porcn & \multicolumn{1}{c}{ frec } & porcn \\
\hline letra h & urb & 5243 & 21.44 & 19211 & 78.56 & 24454 & 100.0 \\
& rur & 1574 & 26.20 & 4433 & 73.80 & 6007 & 100.0 \\
fon /b/ & urb & 6226 & 11.16 & 49579 & 88.84 & 55805 & 100.0 \\
& rur & 2053 & 24.95 & 11684 & 85.05 & 13737 & 100.0 \\
fon /j/ & urb & 861 & 5.29 & 15420 & 94.71 & 16281 & 100.0 \\
& rur & 301 & 8.61 & 3196 & 91.39 & 3497 & 100.0 \\
fon /y/ & urb & 2687 & 4.40 & 58424 & 95.60 & 61111 & 100.0 \\
& rur & 864 & 6.15 & 13195 & 93.85 & 14059 & 100.0 \\
fon /s/ & urb & 6047 & 3.31 & 176742 & 96.69 & 182789 & 100.0 \\
& rur & 2170 & 4.44 & 46716 & 95.56 & 48886 & 100.0 \\
\hline totales & urb & 21064 & 6.19 & 319376 & 93.81 & 340440 & 100.0 \\
& rur & 6962 & 8.08 & 79224 & 91.92 & 86186 & 100.0 \\
\hline núm. txt. & urb & 3131 & & & & & \\
& rur & 915 & & & & &
\end{tabular}

TABla 9

Alogramas: lapsogramas y ortogramas

Niveles socioeconómicos alto, medio, bajo

\begin{tabular}{llrrrrrr}
\hline & & \multicolumn{2}{c}{ lapsogramas } & \multicolumn{2}{c}{ ortogramas } & \multicolumn{2}{c}{ alogramas } \\
& & \multicolumn{1}{c}{ frec } & porc & \multicolumn{1}{c}{ frec } & porcn & \multicolumn{1}{c}{ frec } & porc \\
\hline letra $\mathrm{h}$ & alto & 164 & 8.25 & 1823 & 91.75 & 1987 & $100 . \mathrm{C}$ \\
& medio & 4331 & 23.56 & 14049 & 76.44 & 18380 & $100 . \mathrm{C}$ \\
& bajo & 2336 & 23.64 & 7224 & 76.36 & 9560 & $100 . \mathrm{C}$ \\
fon /b/ & alto & 220 & 4.93 & 4243 & 95.07 & 4463 & $100 .($ \\
& medio & 5275 & 12.48 & 36984 & 87.52 & 42259 & $100 .($ \\
& bajo & 2704 & 12.69 & 18606 & 87.31 & 21310 & $100 .($ \\
fon /j/ & alto & 24 & 1.77 & 1330 & 98.23 & 1354 & $100 .($ \\
& medio & 726 & 5.89 & 11600 & 94.11 & 12326 & $100 .($ \\
& bajo & 401 & 7.07 & 5271 & 92.93 & 5672 & $100 .($ \\
fon /y/ & alto & 81 & 1.91 & 4168 & 98.09 & 4249 & $100 .($ \\
& medio & 2274 & 4.88 & 44316 & 95.12 & 46590 & $100 .($ \\
& bajo & 1163 & 5.06 & 21810 & 94.94 & 22973 & $100 .($ \\
fon /s/ & alto & 228 & 1.72 & 13026 & 98.28 & 13254 & $100 .($ \\
& medio & 5172 & 3.62 & 137646 & 96.38 & 142818 & $100 .($ \\
& bajo & 2710 & 3.85 & 67742 & 96.15 & 70452 & $100 .($ \\
\hline totales & alto & 717 & 2.83 & 24590 & 97.17 & 25307 & $100 .($ \\
& medio & 17778 & 6.78 & 244595 & 93.22 & 262373 & $100 .($ \\
& bajo & 9314 & 7.17 & 120653 & 92.83 & 129967 & $100 .($ \\
\hline núm. txt. & alto & 195 & & & & & \\
& medio & 2419 & & & & & \\
& bajo & 1348 & & & & &
\end{tabular}


GrÁFICA 1

Lapsogramas en primaria porcentajes por categoria

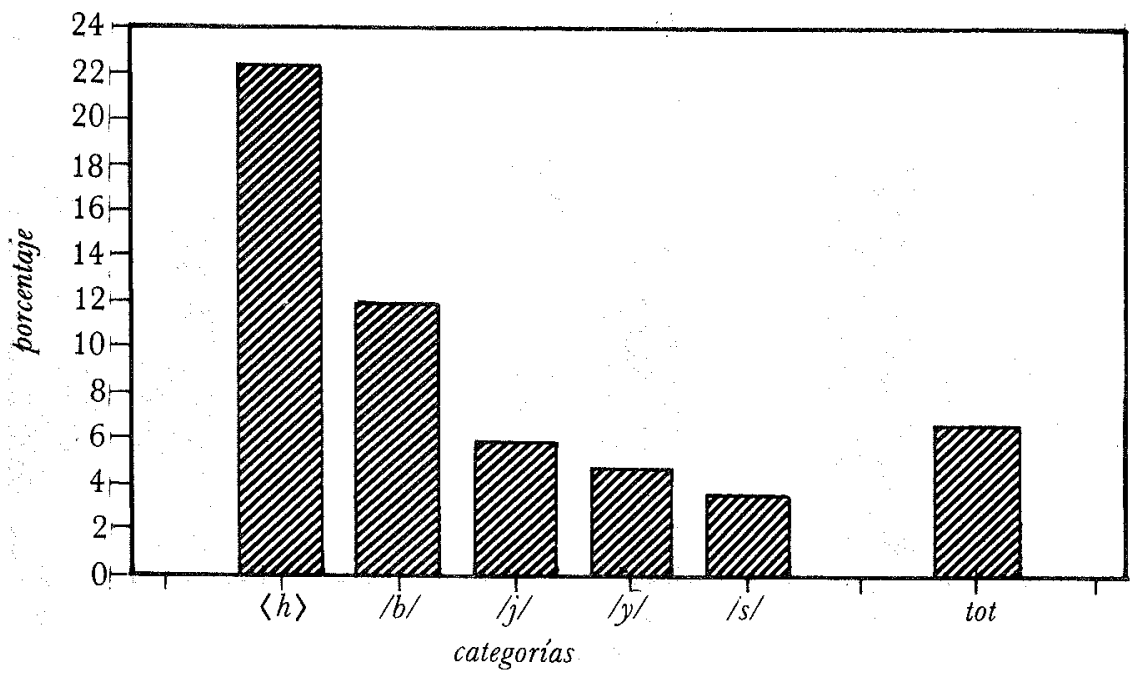

GRÁFICA 2

Lapsogramas en primaria porcentajes grados $3^{\circ}$ a $6^{\circ}$

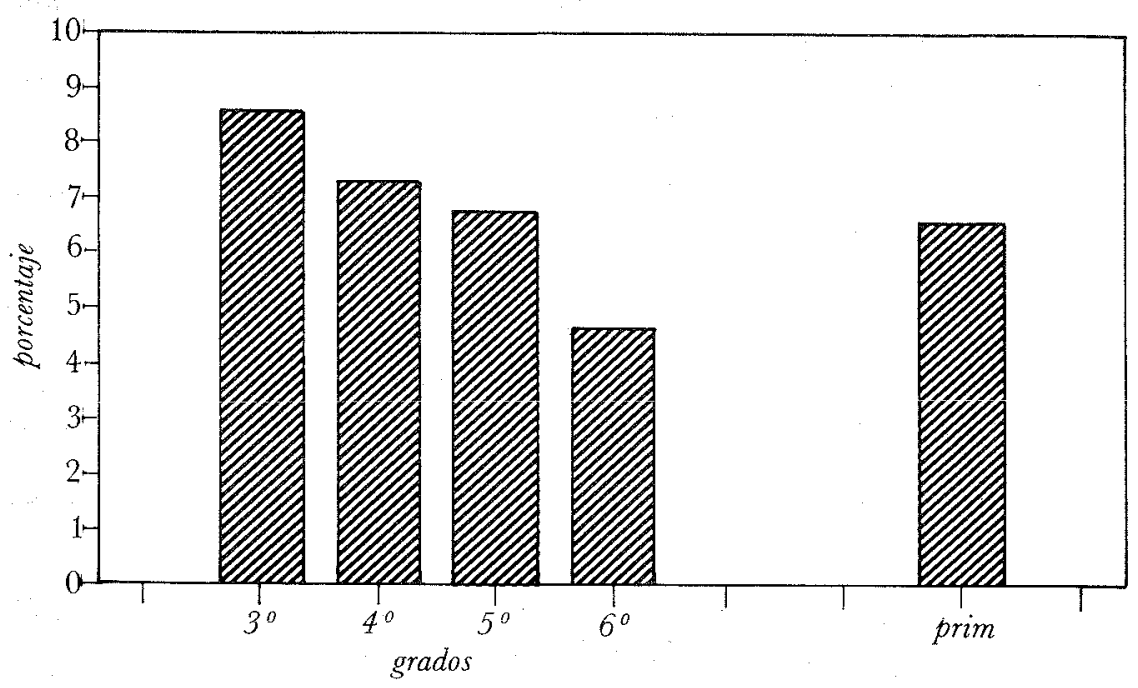


GRÁFICA 3

Lapsogramas en primaria porcentajes por sexo, zona y nivel socioeconómic

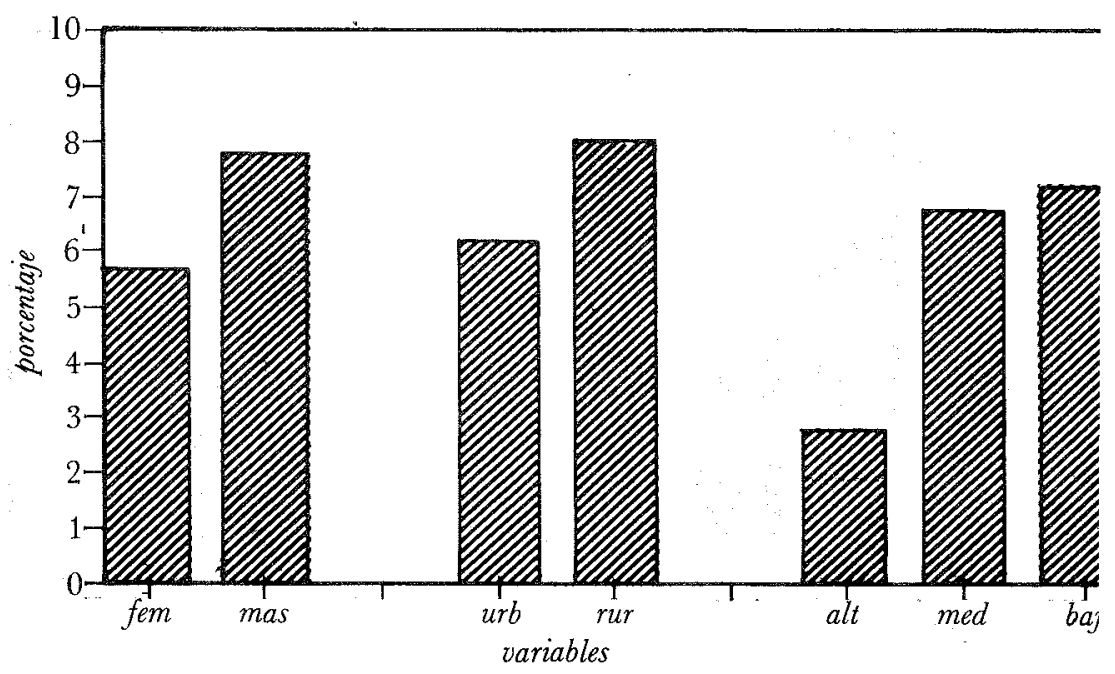

\section{BIBLIOGRAFÍA}

Aaltio, Maija-Hellikki 1976. Finnish for Foreigners. T. 1. $9^{\text {a }}$ ed. Helsink Academia Cubana 1960. "Proyecto de reforma de la ortografía", Tercer $C_{t}$ greso de Academias de la Lengua Española: Actas y Labores, Bogotá, julio 27-ago 6 1960. Academia Colombiana de la Lengua, Bogotá, pp. 265-275. Acordo ortográfico da língua portuguesa 1990. Lisboa, 14-16 Dezembro.

Alarcos Llorach, Emilio 1968. "Les répresentations graphiques du là gage", en Le langage. Gallimard, Paris, pp. 513-568.

AlcalÁ, Antonio 1983. "La reforma ortográfica de Andrés Bello", Homer je a Andrés Bello: memoria. UNAM, México, pp. 75-107.

Applebee, Arthur N., Judith A. Langer, \& Ina V. S. Mulle 1988. I1 reads best? Factors Related to Reading Achievment in Grades 3, 7 and 11. Edu tional Testing Service, Princeton, NJ.

ÁviLA, RaÚL 1974. "Problemas de fonología dialtectal", NRFH, 23, 369-3؛

- - (ed.) 1982. Así escriben los niños de México. SEP-Comisión del Idioma ] pañol, México.

— , 1984a. "Ortografía y estrato social", VII Congreso de la Asociación de L güistica y Filología de la América Latina. Santo Domingo, República Domi cana [en prensa].

—, 1984b. "Para que nos comprendan los niños", Revista de Computac 010, México, núm 5.

— 1985 . "El análisis de textos infantiles por computadora: resultado posibilidades", Segundo Simposio Internacional "La computación y la educac infantil'. Actas. CONACYT-SEP-FAR, México, pp. 8.1-8.14

, 1986a. "Léxico infantil de México: palabras, tipos, vocablos", Actas $d t$ Congreso Internacional sobre el Español de América. UNAM, México, pp. 510-5 
_ـ 1986b. "Sólo para tus oídos: ejercicio de lectura en voz alta a propósito de una reforma de la ortografía española", en L. F. Lara, y F. Garrido (eds.), Escritura y alfabetización. El Ermitaño, México, pp. 11-38.

—, 1988. "Análisis de textos escritos en población escolar mexicana", en A. Ardila, y F. Ostrosky-Solís (eds.), Lenguaje oral y escrito. Trillas, México, pp. 194-206.

— , 1989. "Un alfabeto fonológico práctico para el español: pros y contras", $A L M$ [en prensa].

—, et al. 1980. Mi libro de primero. 4 ts. SEP, México.

—, et al. 1981. Mi libro de segundo. T. 1: Ejercicios, t. 2: Lecturas. SEP, México.

— - et al. 1982. Español tercer grado. T. 1: Ejercicios, t. 2: Lecturas. SEP, México.

BALDI, P. L. 1972. "Fattori sociali dell'abilità linguistica nella produzione scritta di bambini di nove-dieci anni", SILTA, 1, núm. 3, 335-471.

BeCK, M. 1988. "Can't spell? Yur not dumm", Newsweek, June 6, 48 ss.

BELLO, A. 1823. "Indicaciones sobre la conveniencia de simplificar y uniformar la ortografía en América", Obras completas. Ministerio de Educación, Caracas, 1951, t. 5, pp. 69-87 [ $1^{\text {a }}$ ed. en la Biblioteca Americana, Londres, 1823]:

- 1844. "Ortografía", Obras completas. T. 5, pp. 97-115 [1 $1^{\text {a }}$ ed. en El Araucano, Santiago de Chile, 1844].

Bernstein, Basil 1971. Class, Codes and Control. Ts. 1 y 2. Routledge \& K. Paul, London.

—, 1974 . "Códigos amplios y restringidos: sus orígenes sociales y algunas consecuencias", en P. Garvin, y Y. Lastra de Suárez (eds.), Antología de estudios de etnolingüistica y sociolingüistica. UNAM, México, pp. 357-374.

Bravo Valdivieso, L. 1988. "Las dislexias: investigación en Latinoamérica", en A. Ardilla, y F. Ostrosky-Solís (eds.), Lenguaje oral y escrito. Pp. 172-193.

Brown, L. A., A. S. Palincsar, \& L. Purcel 1985. "Poor Readers: Teach, don't label', en Neisser (ed.), The Adacemic Performance in Minority Children: A New Perspective, Erlbaum, Hillsdale, NJ.

Conseil Supérieur de la Langue Française 1990. "Les rectifications de l'orthographe", Journal Officiel de la Republique Fraņ̧aise, núm. 100, 6 décembre.

Downing, J., y R. Flores 1975. "La relación que existe entre la dislexia y el sistema de escritura usado en el idioma", Memoria del II Congreso Hispanoamericano de Dificultades en el Aprendizaje de la Lectura y la Escritura. Dirección Gral. de Educación Especial, SEP, México, pp. 685-691.

Ferreiro, E. 1986. "La complejidad conceptual de la escritura", en L. F. Lara, y F. Garrido (eds.), Escritura y alfabetización. Pp. 60-81.

Gardner, Howard 1978. Developmental Psychology: An Introduction. Little, Brown \& Co., Boston-Toronto.

Gardner, H., D. Wolf, \& A. Smith 1975. "Artistic Symbols in Early Chilhood", New York Univ. Educational Quarterly, t. 6, pp. 13-21.

Greenhouse, S. 1991. "In Le Française, it will be huitres instead of hûitres", The New York Times, June 23.

LARA, L. F. 1986. "La normatividad en la ortografía", en L. F. Lara, y F. Garrido (eds.), Escritura y alfabetización. Pp. 82-101.

Mosterín, Jesús 1981. La ortografía fonémica del español. Alianza Editorial, Madrid. 
Nebrija, Antonio de 1492. Gramática de la lengua castellana. Ed. y est. de 1 Quilis. Editora Nacional, Madrid, 1980.

Negrete, J. 1985. "Por qué Juanito no aprende ortografía", Segundo Simp sio Internacional "La computación y la educación infantil". Actas. CONACY'. SEP-FAR, México, p. 68.1.

Negrete, J., y E. Ariza 1987. "Por qué Juanito no aprende ortografía' Revista de Computación 010, México, núm. 6, p. 31.

O'Dogherty, LaUra 1983. El lenguaje como factor del rendimiento escolar. Tesi Instituto Tecnológico Autónomo de México, México.

Olivieri, C. 1990. "Ortographe: la fin d'un tabou", Le Français dans Monde, núm. 236.

Polo, José 1974. Ortografía y ciencia del lenguaje. Paraninfo, Madrid.

Rodríguez López, Marina 1986. La teoría de los códigos lingüísticos de Ba Bernstein: una aplicación práctica. Tesis, Universidad de La Laguna, C narias.

Rosenblat, Á. 1951. "Prólogo", en Andrés Bello, Obras completas. T. pp. i-clx.

Schmid, M. A. 1985. "Cuestiones ideológicas y problemas prácticos de enseñanza de la escritura en la lengua materna", en Lingüística y enseñan de la lengua materna. UNAM, México, pp. 123-137.

Terman, L. M. \& L. E. Tyler 1954. "Psychological Sex Differences", L. Carmichael (ed.), Manual of Child Psychology. $2^{\mathrm{a}}$ ed. Wiley, New Yor pp. 1064-1114.

UngeHEUER, F. 1991. "How do you spell outrageous?", Time, January 1 pp. 33 ss.

VÁzQUez, María Eugenia 1989. La práctica de la redacción en la escuela primar los mensajes de los niños a través de textos escritos. Tesis, UNAM, México. 